\title{
MIXITÉ À LA FRANÇAISE
}

Une vision politique de la ville lissée

Jean-Pierre Lévy

\author{
La Découverte | « Mouvements »
}

2006/5 nº 47-48 | pages 167 à 173

ISSN 1291-6412

ISBN 2707149403

Article disponible en ligne à l'adresse :

http://www.cairn.info/revue-mouvements-2006-5-page-167.htm

\section{Pour citer cet article :}

Jean-Pierre Lévy, "Mixité à la française. Une vision politique de la ville lissée », Mouvements 2006/5 ( $\mathrm{n}^{\circ}$ 47-48), p. 167-173.

DOI 10.3917/mouv.047.0167

Distribution électronique Cairn.info pour La Découverte.

(C) La Découverte. Tous droits réservés pour tous pays.

La reproduction ou représentation de cet article, notamment par photocopie, n'est autorisée que dans les limites des conditions générales d'utilisation du site ou, le cas échéant, des conditions générales de la licence souscrite par votre établissement. Toute autre reproduction ou représentation, en tout ou partie, sous quelque forme et de quelque manière que ce soit, est interdite sauf accord préalable et écrit de l'éditeur, en dehors des cas prévus par la législation en vigueur en France. Il est précisé que son stockage dans une base de données est également interdit. 


\section{Mixité à la française Une vision politique de la ville lissée}

\section{PAR}

Jean-PierRe Lévy*
La notion de mixité est aujourd'hui largement employée dans les textes et les discours de la politique de la ville en France. De nombreux travaux interrogent aujourd'hui la validité du concept, notamment dans ce qu'il peut avoir d'effets pervers dans les politiques de peuplement menées au niveau local. Pour mettre en évidence les sens et représentations de la ville portés par le discours politique, il est nécessaire de revenir sur la construction politique des notions de mixité et d'équilibre social, sur l'histoire de leur émergence dans les textes législatifs français contemporains.
$\mathrm{L}$ a "mixité sociale ", comme objectif politique du peuplement des quartiers défavorisés, est un leitmotiv, voire une catégorie, de l'action publique contemporaine. C'est aussi une notion floue par essence, rarement définie, notamment par les politiques et les différents textes législatifs qui s'y réfèrent. Au sein du "monde social ", de nombreuses voix s'élèvent aujourd'hui pour contester la légitimité ou la validité de la référence à la " mixité sociale " pour organiser les politiques de l'habitat ou justifier les actions menées envers telle ou telle catégorie de population. Au-delà de ce débat très vivant entre partisans et réfractaires à la notion, il peut être utile de sortir de cette opposition pour s'interroger sur le sens de la référence récurrente à la mixité sociale dans la politique de la ville et la gestion du peuplement en France. Il ne s'agira donc pas ici de tenter une évaluation des politiques de " mixité sociale ", ni même d'analyser ses effets pervers, mais d'essayer de mettre en évidence ce que la référence véhicule comme représentations plus ou moins mythiques de la ville, portées par la sphère politique. Vision dont on fait l'hypothèse qu'elle est révélatrice du sens caché, volontaire ou mécanique, des politiques de "mixité sociale".

\section{- De la gestion de la pauvreté à l'équilibre social}

Si les problématiques de la coexistence spatiale et de son corollaire, la ségrégation, préoccupent nombre de chercheurs sur l'urbain au début des années 1970, elles ne constituent pas encore un véritable enjeu pour un milieu politique préoccupé par la résolution de la crise du logement et l'insalubrité du parc immobilier français. Cette indifférence du milieu politique cessera cependant quelques années plus tard à la faveur d'éléments fortement associés, qui auront des répercussions à la fois sur les processus de peuplement des logements sociaux et sur leur gestion politique.

\footnotetext{
* Géographe.
} 
Le premier de ces éléments concerne la politique de regroupement familial menée lors du septennat de Valéry Giscard d'Estaing. Elle va dans un premier temps contribuer à ethniciser la question du logement, puis dans un second temps participer à un glissement des termes du débat, qui bascule de la gestion sociale vers la gestion spatiale. Ce mouvement aboutira, au début des années 1980, lors de l'arrivée de la gauche au gouvernement, à la mise en place d'une politique de la ville qui sera prolongée jusqu'à aujourd'hui.

À l'origine, l'ethnicisation de la question du logement provient des problèmes posés par la présence de travailleurs immigrés dans l'habitat vétuste des quartiers anciens de banlieue, et de leur exploitation par des " marchands de sommeil " peu scrupuleux. Très rapidement cependant, elle sera posée comme s'inscrivant dans la question du peuplement des "grands ensembles". Le départ des couches moyennes - présentes dans les premières générations de locataires des logements sociaux, puis attirées par le développement de l'accession à la propriété soutenu par la politique nationale du logement -, le maintien des plus pauvres, feront apparaître, dès le milieu des années 1970, une forte concentration d'immigrés parmi les habitants des cités d'habitat social, notamment dans les villes gérées par le Parti communiste.

Dans ses travaux sur Gennevilliers, une ville gérée par le Parti communiste, Olivier Masclet révèle ainsi qu'un "plan de travail pour la dissémination du pourcentage de travailleurs immigrés " a été proposé par l'équipe municipale dès $1972^{1}$. Ici, la "menace "d'une concentration résidentielle des immigrés a donc été considérée bien avant leur entrée dans les logements sociaux. Ce faisant, et comme le note Olivier Masclet toujours, la précocité des reven- dications des maires communistes leur permet d'acquérir une légitimité dans le débat des années 1980. La dénonciation des marchands de sommeil, qui gagnent leur vie en accueillant dans des conditions de logement déplorables les ouvriers immigrés, se déplace progressivement vers une dénonciation d'une toute autre nature, celle de l'inégalité qui consisterait à faire assumer la gestion de la pauvreté - et celle des travailleurs migrants - par les seules communes qui ont eu le courage de développer une véritable politique locale du logement et de construire de l'habitat social.

Au début des années 1980, l'argumentaire du discours des maires de banlieue est donc déjà très affuté. Il est dénonciateur et s'inscrit dans le prolongement des affrontements État/municipalité des années 1970. Il inscrit la question de l'ethnicisation dans le cadre plus global de la gestion de la pauvreté eu égard aux ressources municipales. Cet argument permet à des municipalités, comme celle de Gennevilliers, de revendiquer et de légitimer la mise en place précoce d'un blocage de l'entrée des immigrés dans les logements sociaux de la ville.

Ainsi, la pression exercée dès 1980 par Lucien Lanternier, maire de Gennevilliers, sur les sociétés anonymes d'HLM, se comprend comme le prolongement d'une stratégie entamée quelques années plus tôt par son prédécesseur Waldeck Lhuillier, mais élaborée à l'origine pour lutter contre le mal logement dont souffraient les immigrés locataires d'une chambre dans des hôtels meublés insalubres. C'est ainsi qu'à cette date, le maire envoyait un courrier à l'OCIL,

1. O. Masclet, "Une municipalité communiste face à l'immigration algérienne et marocaine. Gennevilliers 1950-1972 ", Genèses, n 45, décembre 2001. 
organisme collecteur du $1 \%$ patronal qui, via ses sociétés anonymes, attribuait nombre de logements sociaux à Gennevilliers. Dans ce courrier, il dénonce la politique d'immigration du patronat et ses conséquences sur le peuplement des communes de banlieue. Le maire s'appuie sur la politique de constructions de logements sociaux de la ville engagée pour lutter contre l'insalubrité du parc ancien, afin de légitimer une injonction au blocage de l'entrée des immigrés dans les logements sociaux de la ville.

"Pouvoir et patronat pratiquent depuis des années une politique d'immigration inhumaine: faisant venir des travailleurs des pays lointains, les exploitant durement dans leur travail, ils leur assurent le plus souvent de mauvaises conditions de logement et ils soubaitent transformer des villes ouvrières en véritables ghettos réservés aux familles les plus pauvres. [...] La ville de Gennevilliers a contribué à mettre un terme aux logements insalubres existant sur son territoire et à assurer le relogement des familles y habitant. [...] La ville de Gennevilliers sur une population de 52000 habitants compte $28 \%$ de travailleurs immigrés. Nous estimons qu'il est injustifié que Gennevilliers, comme Levallois, Colombes ou Nanterre, accueille un tel pourcentage de travailleurs migrants alors que les villes comme SaintCloud, Neuilly, Sceaux, etc., n'en accueillent qu'un nombre extrêmement limité.

Je vous demande donc non seulement de ne plus reloger dans les immeubles gérés par vous à Gennevilliers de nouvelles familles immigrées, et d'étudier leur relogement dans les villes citées précédemment, car ces familles ont droit à un logement décent, mais aussi par le même processus de reloger dans d'autres patrimoines, avec leur accord, un certain nombre de familles actuellement logées à Gennevilliers ${ }^{2}$. "

Il va de soi que, parallèlement à cette injonction, la ville pratiquait elle-même un blocage aux immigrés dans les logements gérés par l'Office municipal d'HLM. Blocage d'ailleurs ouvertement revendiqué en 1984 par Lucien Lanternier dans un entretien à la revue Les Temps Modernes.
"Nous disons nous devons bloquer ll'entrée des étrangers dans le parc sociall dans l'intérêt de tout le monde, bloquer la venue d'une autre série de travailleurs supplémentaires à Gennevilliers, parce que l'on a pas les moyens de faire plus. Sinon on arrivera jamais à faire en sorte que ceux qui sont là, immigrés et français aient des conditions correctes de formation, d'insertion pour une vie meilleure ${ }^{3}$."

Tout d'abord centré sur le problème du mal logement des étrangers concentrés dans de l'habitat vétuste, la dénonciation s'est donc déplacée, dans les années 1980, vers la concentration des travailleurs migrants dans les logements sociaux et la formation de ghettos de pauvres et d'étrangers. Le déplacement du contenu a des conséquences qui vont au-delà du seul positionnement politique ou du blocage de l'accès au parc social aux immigrés. Il aboutit aussi à redéfinir la fonction résidentielle du logement social et de ses représentations dans l'imaginaire collectif. Dans le discours des maires des villes sur lesquelles une grande part de ces logements a été construite, les "HLM " deviennent de ce fait ce qu'ils n'étaient pas à l'origine: le logement des pauvres. Mais bien plus, ce déplacement ouvre également la voie à l'introduction d'une nouvelle notion dans la gestion du logement, inexistante jusqu'alors, celle de "l'équilibre social".

\section{- De l'équilibre à la mixité sociale}

Au moment où la gauche accède au pouvoir en 1981, le discours fait mouche. En cela, publié en 1984, l'entretien de Lucien Lanternier est déjà en retrait. Car à cette date, la prise en compte institutionnelle des revendications des maires de banlieue, souvent communistes, est déjà effective. Dès 1982, la troisième proposition du rapport de la Commission des maires sur la sécurité présidée par Gilbert Bonnemaison (publié en 1983) s'intitulait " Répartir équi-

2. Lettre de Lucien Lanternier à l'OCIL datée du 17 janvier 1980.

3. Les Temps Modernes, Paris, $\mathrm{n}^{\circ}$ 452-453-454, 1984, p. 1666. 
tablement les logements sociaux entre les communes ". Les auteurs demandaient que l'État impose la réalisation de logements sociaux à une collectivité locale, lorsque le rapport du nombre de logements sociaux sur le nombre total de logements de la commune est inférieur à la moyenne régionale.

Cette proposition déplace une nouvelle fois le contenu du débat, en le faisant glisser de la gestion de la pauvreté vers la nécessité d'introduire un équilibre social dans le peuplement au nom du devoir de solidarité. Même si elle figurait déjà dans les textes relatifs à la procédure Habitat et vie sociale (HVS) promulgués en 1977, la référence à l'équilibre social était pourtant restée marginale jusqu'alors. Un an après la publication du rapport Bonnemaison, elle sera reprise dans le rapport Dubedout, dont le chapitre IV s'intitule "Équilibrer la composition sociale des quartiers". Ce rapport nomme les lieux de l'exclusion: essentiellement "les quartiers des grands ensembles ", ainsi que ses formes identifiées par leur composition sociale et la forte proportion d'étrangers. Il légitime au passage le refus des attributions des logements sociaux aux étrangers "qui peut être envisagé en tant [...] que moment d'élaboration d'un plan d'ensemble de composition sociale des quartiers" (p. 53). Ce rapport appelle tout à la fois à la maîtrise des attributions des logements sociaux par les municipalités et à "une politique de rééquilibrage dynamique " qui doit s'inscrire "dans la durée " et "la revalorisation des quartiers" (p. 60).

La notion d'équilibre est en fait empruntée à l'analyse économique, mais, appliquée à la gestion de l'occupation du logement social, la référence au "rééquilibrage "spatial n'est pas dénuée d'ambigüité. Dans une logique de " contrôle étroit des classes populaires " elle implique d'une part une ouverture du parc social à des populations plus aisées que les résidents actuels; mais également une fermeture, en le considérant avant tout comme destiné aux plus pauvres et, dans ce cas, à sa dispersion sur l'ensemble du territoire afin que chacun puisse prendre part aux charges induites par leur ges- tion. Mais personne ne relèvera ce paradoxe et, une fois lancée, la notion de "rééquilibrage " sera utilisée comme telle, à la fois dans les textes législatifs à venir qui la reprendront en l'associant à la diversité puis par conséquent dans le vocabulaire et les objectifs des politiques municipales du logement.

La loi du 31 mai 1990, dite Loi Besson, visant à la mise en ouvre du droit au logement, y fait explicitement référence en évoquant la création des Plans d'occupation du patrimoine social (POPS) et de la "nécessaire diversité de la composition sociale de chaque quartier, de chaque commune et de chaque département, en vue de faire contribuer de manière équilibrée chaque commune au logement des personnes défavorisées". Cette association entre l'équilibre et la diversité sera également reprise un an plus tard dans la Loi d'orientation sur la ville (la LOV). Ce texte stipule que le Programme local de l'habitat vise "à assurer entre les communes et entre les quartiers d'une même commune une répartition équilibrée et diversifiée de l'offre de logements " en instaurant "une participation à la diversité de l'habitat " par la construction de logements sociaux ou le versement d'une taxe.

Pourtant, dans ces différents textes, nulle part le terme de " mixité sociale " n'est mentionné. Il faudra en fait attendre 1998 et la Loi d'orientation relative à la lutte contre les exclusions pour que les termes d'équilibre, de diversité et de mixité soient associés, par exemple en stipulant que "la construction, l'aménagement, l'attribution et la gestion des logements locatifs sociaux [...] contribuent à la nécessaire mixité sociale des villes et des quartiers". On les retrouvera dans la loi Solidarité et renouvellement urbain (SRU) qui impose $20 \%$ de logements sociaux au sein des communes afin "de favoriser la mixité sociale en assurant entre les communes une répartition équilibrée et diversifiée de l'offre de logements".

Il aura donc fallu vingt ans pour que le législateur parvienne à définir la mixité sociale. Implicitement, il s'agit d'instaurer une harmonie sociale, dont il est dit explicitement qu'elle sera obtenue par le recours à la "mixité sociale ", 
conçue comme une méthode pour créer une ville " diversifiée " et " équilibrée " socialement. Dès lors la mixité sociale peut s'interpréter comme ce qui sépare la ville équilibrée de la ville déséquilibrée. Mais le contenu de cet équilibre social restait encore à expliciter...

\section{- Le calcul des écarts}

S'il existe une distance entre l'équilibre et le déséquilibre devant être comblée par l'introduction d'une mixité sociale dans le peuplement, celle-ci devient quantifiable. Logiquement donc, l'objectif d'une mesure du déséquilibre est apparu en phase avec celui de la mise en œuvre de la mixité.

C'est dans cet esprit qu'à la suite de la publication d'une note du Groupe d'étude sur les discriminations (GELD), portant sur les attributions de logements sociaux, Marie-Noëlle Lienemann, secrétaire d'État au Logement, se déclarait intéressée par la création d'un indice de mixité sociale à établir par quartiers, par territoires et par organismes. Cet indice permettrait de "voir comment rétablir les équilibres nécessaires, là où manifestement, les logiques de ségrégation prennent le pas sur l'intégration ${ }^{4}$ ". Dans cette déclaration, la mixité est clairement associée à l'intégration, contre la ségrégation. Mais les indicateurs proposés à l'époque pour la mesurer s'avéreront faibles (revenus, composition du ménage et âge de la personne de référence) et, le gouvernement changeant, l'indice ne verra jamais le jour.

L'initiative aura cependant de l'importance, parce qu'elle définit la mise en ouvre de la mixité sociale à partir du calcul d'un écart entre une situation censée marquer l'équilibre et une autre identifiée comme déséquilibrée. Certes, le recours au calcul des écarts n'est pas vraiment une nouveauté, dans une politique de la ville où la volonté d'utiliser des " indicateurs " pour mettre en évidence des situations "spécifiques " par rapport à une moyenne globale est finalement courante, au risque de fournir une représentation standardisée des quartiers concernés. Mais jusque là, les écarts ne visaient qu'à identifier des quartiers particuliers. Une nouvelle étape est dorénavant franchie, dans la mesure où le politique revendique le calcul des écarts dans le dessein de résoudre les problèmes posés par un "déséquilibre ". On assiste ainsi à une instrumentalisation du calcul des écarts par l'action publique, dans une logique qui voudrait que, si l'on comble ces " écarts " dans une situation " déséquilibrée ", celleci revienne à "l'équilibre ". Mais cette logique offre également une opportunité: celle d'en déduire, grâce à une formulation " institutionnelle " des écarts, la situation urbaine idéale-typique attendue par le politique et, pour la première fois, de définir ce que recouvre une situation urbaine équilibrée socialement.

C'est dans la continuité de la proposition de Marie-Noëlle Lienemann que s'inscrit la Loi sur la rénovation urbaine proposée par Jean-Louis Borloo, ministre délégué à la Ville, en août 2003. L'objectif de cette loi est de réduire les inégalités sociales. Son contenu est explicite: cette réduction passe par le "retour au droit commun" des quartiers sensibles. La notion de " mixité sociale " n'apparaît pas avant l'article 6 de la loi qui annonce la création "d'un programme national de rénovation urbaine visant à structurer, dans un objectif de mixité sociale et de développement durable, les quartiers classés en zone urbaine sensible ". La notion réapparaît dans une même logique dans l'article 10.

Par contre, la notion d'écart est mentionnée d'emblée et le terme est utilisé dans les trois premiers articles de la loi. Car il s'agit bien de "réduire les écarts de développement entre les

4. Le Monde, 27/04/2002. 
territoires ", (article 1), de "tendre à réduire de façon significative les écarts constatés " (article 2) et de "mesurer l'évolution des inégalités sociales et des écarts de développement "(article 3).

Pour ce faire, la loi propose une méthode précise: définir des espaces et des échelles de référence variables (le territoire national, l'agglomération dans laquelle est située le quartier concerné, et même l'ensemble des Zones urbaines sensibles); calculer des indicateurs; mesurer, pour ces indicateurs, les écarts par rapport aux espaces de référence; mener une politique qui sera évaluée au regard de la réduction de ces écarts. À cette fin est créé un Observatoire national des zones urbaines sensibles qui centralise l'information et qui est chargé d'évaluer ces politiques. Une réduction significative des écarts constatés avec les autres villes ou quartiers marquera " le retour au droit commun " de ces quartiers.

Ces indicateurs

participent à construire une représentation formatée et stigmatisante des habitants de ces quartiers, non dénuée parfois de certains relents hygiénistes. entre autres... Mais aussi "le rétablissement de la tranquillité et de la sécurité publique ". L'annexe I de la loi va plus loin, en dressant une liste précise des indicateurs à évaluer, précédée d'une liste des objectifs. Et que trouve-t-on?

Sans une volonté d'exhaustivité, on peut citer pêle-mêle:

- évolution annuelle du taux de chômage dans les ZUS et dans l'ensemble des agglomérations concernées par la politique de la ville;

- évolution annuelle du nombre de demandeurs d'emploi étrangers résidant en ZUS;

- nombre d'entreprises existantes, créées ou transférées; - nombre annuel de logements sociaux réhabilités, construits ou démolis;

- ratio de praticiens médicaux et paramédicaux pour 5000 habitants et nombre d'actes par médecin généraliste;

- taux de réalisation des prescriptions de soins à l'issue des bilans de santé scolaire;

- proportion d'élèves en retard au début et à la fin du cycle 3 , de deux ans ou plus, en $6^{\text {e }}$ et en $3^{\text {e }}$ générale;

- taux de réussite au baccalauréat;

- proportion d'élèves boursiers reçus au brevet des collèges et au baccalauréat;

- atteintes aux personnes et aux biens privés;

- agressions en milieu scolaire;

- trafic de stupéfiants;

- mauvais traitements et abandons d'enfants.

Ces indicateurs se veulent globaux et concernent un large spectre de la vie sociale et économique des individus. Cependant, en globalisant ces différentes dimensions, on fait en sorte qu'ils participent à construire une représentation formatée et stigmatisante des habitants de ces quartiers, non dénuée parfois de certains relents hygiénistes. Ces habitants seraient locataires d'un logement social certes, mais aussi et tout à la fois des étrangers au chômage, des élèves boursiers en échec scolaire, des délin- 
quants et des personnes malades très coûteuses en actes médicaux. Ce que ces indicateurs donnent à voir ce n'est pas tant une réalité sociale, qu'une représentation d'un cumul de pathologies sociales qui éloigneraient ces lieux d'un "droit commun ", lui-même pré-construit par opposition à ces mêmes indicateurs.

Mais au-delà de travers propres à la mise en place d'indicateurs chiffrés à vocation " objective " censés pouvoir décrire une réalité sociale que l'on sait complexe, ce qui est nouveau ici ce sont les objectifs assignés à leur construction et, par là même, à la politique de laquelle ils participent. Dans l'annexe I de la loi, en préambule de la présentation des indicateurs, nous pouvons lire la phrase suivante: " $L a$ politique de la ville se justifie par l'objectif de réduction progressive des écarts constatés avec les autres villes ou quartiers, et de retour au droit commun. "L'objectif ne serait donc pas de résoudre ce qui relèverait de ces pathologies, mais de faire en sorte que, réduites au rang d'indicateurs, elles entrent dans les fourchettes codées d'un droit commun définissant une situation moyenne. L'objectif ne serait donc pas tant la résolution des problèmes, ou ce qui est identifié comme tels, que leur transparence en ces lieux.

Par là même, "l'écart " désigne les pathologies et les responsables du " déséquilibre " (les logements sociaux, les chômeurs, les étrangers sans travail, les jeunes en échec scolaire, les délinquants...). La " mixité sociale " devient le moyen de rendre ce "déséquilibre " ou ces pathologies invisibles dans l'espace concret et statistique. Le "droit commun " quant à lui est l'objectif à atteindre, c'est-à-dire la production d'une ville lissée dans les différents aspects de la vie sociale, la volonté d'un retour à une ville épurée de ses pathologies.

Ce que montre l'évolution de la référence à la "mixité sociale " dans la politique de la ville et les politiques locales, c'est finalement le passage de la gestion de la pauvreté vers la recherche d'un équilibre social mythique. C'est le sens même de la mixité qui a été peu à peu défini et affiné par la sphère politique. Aujourd'hui, la mixité est un moyen de diminuer les écarts entre les bonnes et les mauvaises situations socio-spatiales afin d'agir non pas sur les causes, mais sur la visibilité spatiale d'un " déséquilibre social ". En distinguant les bons des mauvais quartiers, on en vient alors à une représentation socialement lissée de la ville, qui justifie le retour à un "droit commun ". Erigé en catégorie de l'action publique, ce " droit commun " est défini par une batterie d'indicateurs de plus en plus larges et complexes, qui participent d'un contrôle statistique de tous les aspects de la vie sociale des citadins vivant dans des espaces considérés comme non conformes par la puissance publique. 